f. Fragmente. Die erste Clemensausgabe, die auch Fragmente enthält, ist die Potters. Seine Sammlung besteht aus drei Teilen, den Fragmenten, die J. Fell im Anhang zu seiner Ausgabe von Quis div. salv. $1683 \mathrm{~S} .144 \mathrm{ff}$ veröffentlicht hatte, den von Ittig, Opp. Clem. Alex. Suppl. 1760 S. $150 \mathrm{ff}$ und Praef. a $8 \mathrm{ff}$, gesammelten Stücken und drittens aus dem von E. Grabe für sein Spicilegium gesammelten, aber nicht veröffentlichten Material. Dazu kam noch ein Stück, auf das Fabricius, Bibl. graec. V p. 111 hingewiesen hatte. Eine lateinische Übersetzung der hier nur griechisch gedruckten Stücke und einige neue Fragmente brachte dann Fabricius in seiner Ausgabe des Hippolyt II (Hamburg 1718) S. 66 -74. Diese Stücke wurden abgedruckt in dem Nachdruck der Pottersehen Ausgabe von 1757 und bei Gallandi, Bibl. vet. patr. Venet. 1766. 2. Aufl. 1788 II S. 153-159. Die Ausgabe von Migne fügte noch dazu die Fragmente aus der Catenensammlung Cramers. Alle Fragmente wurden gesichtet und neu herausgegeben von Th. Zahn, Suppl. Clem. S. 32-78. Vgl. ferner Barnard a. a. 0. S. 47-52 und Preuschen bei Harnack, Überlief. S. 299-327.

Ein bisher unbekanntes Fragment aus den Hypotyposen veröffentlichte Mercati in den Studi e Testi XII (1904) S. 1-15 aus Vatic. gr. 354. Vgl. dazu A. Harnack, Sitzungsber. d. k. preuss. Ak. d. W. 1904 Nr. 27 S. 901-908.

Die Vermutung, daß der Canon Muratori ein Fragment der Hypotyposen sei, suchte neuerdings Chapman, Revue Bénédictine 21 (1904) S. 240-264 (vgl. auch S. 369-374) wahrscheinlich zu machen. Vgl. aber die scharfe Ablehnung dieser Hypothese durch A. Harnack, Theol. Literaturztg 29 (1904) Sp. 636 f.

\title{
E. Übersetzungen.
}

\section{Die lateinische Übersetzung.}

In der Geschichte des gedruckten Textes spielt eine wichtige Rolle die lateinische Übersetzung. Denn diese ist nicht nur sehr oft selb-

Das zweite Gedicht, das in FM ohne Überschrift auf diesen Hymnus folgt

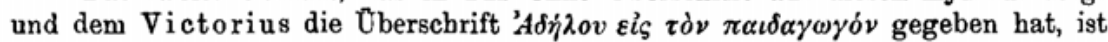
das Parallelgedicht zu dem Hymnus, verfaßt von einem Leser dieses Hymnus. Ich habe daher das Gedicht an den Schluß der Scholien S. 339f gestellt. Ob Arethas der Verfasser ist, wie ich in den Unters. üb. d. Scholien z. Cl. Al. S. 48 vermutete, bleibt zweifelhaft, da FM, die unabhängig von einander auf die Originalschrift des Arethas in $P$ zurückgehen würden, mehrere Fehler (vgl. V. 5. 13. 15. 20. 21) gemeinsam haben. 
ständig erschienen und hat somit die Kenntnis unseres Autors verbreitet, sondern sie ist auch von 1616 an allen Ausgaben, mit Ausnahme der von Klotz und Dindorf, beigegeben worden. Es verlobnt sich daher, auch ihrer hier kurz zu gedenken.

Schon auf der letzten Seite der Editio princeps des griechischen Textes wurde vom Drucker eine lateinische UUbersetzung (a viris doctissimis, qui summo studio laboreque tibi navarunt operam) angekündigt und ein Jahr später erschien die Ausgabe: „Clementis Alexandrini omnia quae quidem extant opera, nunc primum e tenebris eruta Latinitateque donata, Gentiano Herveto Aurelio interprete. Laur. Torrentinus Ducalis typographus excudebat Florentiae 1551." Vier besonders numerierte Teile: I. Widmung an Rodulphus Pius vom 31. Dez. 1549; Protr. mit Scholien (aus Mut. III. D. 7) 47 S. II. Widmung an Hangest von Noyon vom 10. Juli 1549; Paed. I-III mit Scholien und Hymnus auf Christus 113 S. III. Widmung an Cosimo de' Medici vom 6. Juli 1551; Strom. I-VIII. $277 \mathrm{~S}$. IV. Sieben verschiedene Indices $50 \mathrm{~S}$. Die oft wiederholte Angabe, daß die Stromata von Chiriaco Strozzi († 1569) übersetzt seien, geht zurück auf Papyrius Masson (Elog. II p. 229) und Giulio Negri, Istoria degli scrittori Fiorentini (1722) p. 125. Hier heißt es von Strozzi: vertit etiam e Greca in Latinam linguam Clementis Alexandrini Stromatum libros 8, impressos Florentiae a Torrentino. Mit Recht scheint mir diese Nachricht bestritten in den Annali della tipografia Fiorentina di Lorenzo Torrentino ${ }^{2}$ (1819) p. 139, da sonst der Widmungsbrief des Gentianus Hervetus an Cosimo de Medici unverständlich wäre.

Die Übersetzung ist mit großer Eile gemacht worden. Hervetus wurde, wie sein Neffe im Vorwort der Ausgabe von 1590 erzählt, von seinem Verleger so gedrängt, „ut diebus singulis tantum vertere cogeretur, quantum quivis alius calamo potuisset excipere, quo commodius typographiariis operis satisfaceret". Diese Eile merkt man der Übersetzung nur zu sehr an. Heinsius hat in der Vorrede seiner Ausgabe 1616 einige Beispiele dafür, wie falsch Hervetus oft übersetzte, mitgeteilt. Vielfach ist die lateinische Übersetzung ebenso unverständlich wie der griechische Text. Ein Beispiel mag genügen: Die Worte Mivo $\tau \dot{\partial} \nu$

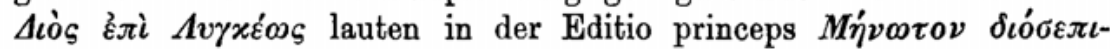

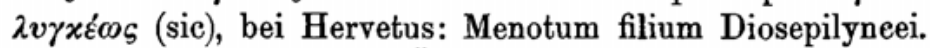

Trotzdem wurde die Übersetzung oft abgedruckt: Basel 1556 Fol.; 1566 Fol.; Paris 1566 Oct.; 1572 Fol. Eine Neubearbeitung erschien 1590: „J. Flavii Clementis opera omnia ... in Latinum conversa, nune vero recognita interpretatione, amplissimis commentariis illustrata a Gentiano Herveto Aureliano ecclesiae Rhemensis Canonico Parisiis 1590 
apud Sebastianum Nivellium." Folio. Die Ausgabe ist besorgt von dem Arzte Simon Hervetus, dem Neffen des Gentianus; derselbe erzählt in dem Widmungsbrief, daß sein Onkel als Achtzigjähriger die Beschäftigung mit Clemens wieder aufgenommen, fast eine ganz neue Übersetzung gemacht und dazu einen Commentar verfaßt habe. Dieser Commentar, der sich mehr durch Wortreichtum als durch tiefen Gehalt auszeichnet, ist in der Potterschen Ausgabe wieder abgedruckt. Außerdem enthielt jene Ausgabe von 1590 p. 769-830: In J. Flavii Clem. Alex. Protr. Paed. Strom. collectanea ex variis doctorum virorum lectionibus, commentariis adversariisque excerpta. Das Meiste davon hat Potter zu den einzelnen Stellen mit dem Sigel Coll. abdrucken lassen.

Ein Titelneudruck scheint die Ausgabe von 1612 (Parisiis ex officina Nivelliana) zu sein; wenigstens stimmen die Ausgaben außer auf den ersten Blättern genau überein. Hervets Übersetzung wurde dann, mehr oder weniger verändert, in den Clemensausgaben von 1616, 1629, 1641, 1688, 1715, 1757, 1778/79, 1857 mit dem griechischen Text gedruckt, außerdem (zusammen mit der Übersetzung von Quis div. salv. durch Combefis und den Adumbrationen) nach der Ausgabe von Heinsius in der Max. bibl. vet. patr. Lugd. 1677 III p. 1-234.

Über den Abdruck des lateinischen Teils der Potterschen Ausgabe vgl. oben S. LXX Anm. 2. Unvollständig ist die lateinische Übersetzung auch enthalten in der von Caillou und Guillon herausgegebenen Collectio selecta SS. ecclesiae patrum Parisiis 1829 III p. 113-482; IV p. 1-386. Auszüge aus Paed. Protr. Strom. I-VII enthält Franc. Rous, Mella Patrum Londini 1650 p. $149-239$.

\section{2. Übersetzungen in moderne Sprachen.}

Anhangsweise seien noch die deutschen, französischen, englischen Übersetzungen der Gesamtwerke oder einzelner Teile besprochen.

1. Deutsch. Eine vollständige Übersetzung gibt es nicht. Auszüge enthält Chr. Fr. Rößler, Bibliothek der Kirchenväter Leipzig 1776 II S. 6-75. In der Kemptener Bibliothek der Kirchenväter ist enthalten Quis div. salv. und Protr., übersetzt von L. Hopfenmüller, und Paed. I-III, übersetzt von J. Wimmer, Kempten 1875. Die Exc. ex Theod. wurden übersetzt von G. Arnold, Unparth. Kirchen- u. Ketzerhistorie Frankfurt 1699. 1700 IV S. 41-50. Gegen ihn schrieb H. G(eorg) W(achter) E(cclesiastes) M(emmingensis), Augenscheinliche Erweisung, daß der vermessene Ketzerpatron Gottfried Arnold das valentinianische Ketzerfragmentum Theodoti weder verständlich noch treulich übersetzet und damit seine schlechte Wissenschaft in der griech. Sprache selbst verraten habe. Ulm 1701. 
Öfters übersetzt wurde der Hymnus auf Christus: von Fr. Münter, Über die älteste christl. Poesie (vor seiner Übersetzung der Offenb. St. Joh.) Kopenhagen 1806 S. 33 ff; von Rambach, Eylert, Schulthess in den oben S. LXXVI angeführten Werken; C. R. Hagenbach in Rheinwalds Repertorium 14 (1836) S. $114 \mathrm{f}$ und Die christl. Kirche in den ersten drei Jahrh. Leipzig 1853 S. 222 (abgedruckt bei J. Kayser, Beiträge z. Geschichte u. Erklärung der ältesten Kirchenhymnen² Paderborn 1881 I S. $28 \mathrm{f}$ und bei 0 . Bardenhewer, Geschichte der altkirchl. Literatur II Freib. i. Br. 1903 S. 32); C. Fortlage, Gesänge christl. Vorzeit Berlin 1844 S. 38-40 (abgedruckt bei A. Baumgartner, Geschichte der Weltliteratur IV Freib.i. Br. 1900 S. 18 f); J.F.H. Schlosser, Die Kirche in ihren Liedern Mainz 1852 II S. 107-109; F. Ranke in F. Pipers Evang. Kalender f. 1868 Berlin 1868 S. 29-31.

2. Französisch. Protr. wurde übersetzt von D. Cousin Paris 1684. - Nur Quis div. salv. und Paed. I-III sind enthalten in: Les Oeuvres de S. Clément d'Alexandrie traduites du Grec [par Nic. Fontaine] Paris chez Pralard 1696. - Auszüge aus allen Werken bietet D. M. N. S. Guillon, Bibliothèque choisie des pères de l'Église greeque et latine Paris 1822 I p. $375 \mathrm{ff}$. - Eine vollständige Übersetzung gab Ant. Eug. de Genoude, Les pères de l'Église IV V Paris 1839.

3. Englisch. Sämtliche Werke sind übersetzt in A. Roberts und J. Donaldson, Ante-Nicene Christian Library vol. IV. XII. XXII S. 185-217; XXIV S. 137-181 Edinburgh 1867-1872. Der Übersetzer war W. Wilson. Das Gleiche bietet der von A. Cleveland Coxe besorgte amerikanische Abdruck der englischen Sammlung: The AnteNicene Fathers II S. 171-604 Buffalo 1887. - Quis div. salv. übersetzte J. Jones (Clement, A discourse concerning the salvation of rich men London 1711) und P. Mordaunt Barnard (in der Sammlung Early Church Classics, herausgegeben von der Society for Promoting Christian Knowledge London 1901). - Einige ausgewählte Stäcke aus Clemens übersetzte R. Ornsby, The Month 19 (1873). - Joseph B. Mayor begleitete seine Ausgabe von Strom. VII mit einer englischen Übersetzung London 1902.

\section{F. Bemerkungen zu der vorliegenden Ausgabe.}

Die erste Anregung zur Beschäftigung mit Clemens Alex. erhielt ich im Jahre 1888 durch Herrn Geheimrat Dr. Iwan von Müller, damals Professor in Erlangen, der mir riet, eine Arbeit über diesen Autor für das philologische Specialexamen einzureichen und mich nicht nur bei Anfertigung jener Arbeit (sie wurde als Doctordissertation gedruckt: 